\title{
LES SÉVICES COMMIS PAR LA FAMILLE DESGROTTES, HISTOIRES DE MALTRAITANCE D'ESCLAVES ET DE LEUR RÉSISTANCE À LA MARTINIQUE
}

\author{
LAS SEVICIAS COMETIDAS POR LA FAMILIA \\ DESGROTTES. HISTORIAS DE MALTRATO DE \\ ESCLAVOS Y SU RESISTENCIA EN MARTINICA
}

\author{
ULRIKe SCHMIEDER \\ Leibniz Universität Hannover
}

\section{RESUMEN}

Esta contribución cuenta una micro-historia partiendo de un caso de sevicia contra esclavos revelado por un periódico abolicionista e investigado por el Estado colonial en los últimos años de la esclavitud en Martinica. Mira el comportamiento general de la familia propietaria de esclavos y la resistencia de las esclavas maltratadas y otros esclavos alrededor de ellas. El papel reconstruye las familias de los libertos en el momento de emancipación y los conflictos sociales en las plantaciones de la familia Desgrottes después de la abolición. Finalmente, investiga la herencia de los conflictos raciales-sociales de la esclavitud y post-esclavitud hasta hoy.

Palabras clave: Martinica, esclavitud, post-emancipación, resistencia, insurrección esclava.

\begin{abstract}
This contribution counts a micro-history starting at a case of bad treatment of slaves revealed by an abolitionist newspaper and investigated by the colonial state in the final years of slavery in Martinique. It looks on the general behavior of the slave owner's family and the resistance of mistreated slave women and their families and other slaves around. The paper reconstructs the family of the freedmen in the moment of emancipation and the social conflicts on the plantations of the family Desgrottes after abolition. Finally, it investigates the heritage of the racial-social conflicts of slavery and post-slavery until today.
\end{abstract}

Keywords: Martinique, slavery, post-emancipation, resistance, slave insurrection. 


\section{L'esclavage en Martinique}

La France annexa l'île de la Martinique en 1635. Quelques années plus tard éclatait la révolution de la canne à sucre. ${ }^{2}$ Au cours des XVllème et XIXème siècles, environ 217.000 Africains furent déportés en Martinique ${ }^{3}$ pour produire de la canne à sucre sur les habitations. ${ }^{4}$ Aux XVllème et $\mathrm{XVIllème} \mathrm{siècles,} \mathrm{il} \mathrm{s'agissait} \mathrm{«des} \mathrm{Angolas} \mathrm{(Bantous} \mathrm{embarqués} \mathrm{du} \mathrm{royaume}$ d'Angola)», "des Sénégals (Wolofs, Sereres, esclaves des Maures et même Bambaras)» et des "Aradas (vendus par le Roi de Ardres)», ces derniers formaient le groupe le plus important (GAUTIER: 1985, p. 37).

Le Code Noir de 1685 réglait l'esclavage sur l'île et toute la Caraïbe française; il contenait quelques propositions pour protéger les esclaves de la violence des propriétaires et leur garantir l'alimentation, ainsi qu'un grand nombre de règlementations réglant la répression des esclaves, dont des actes barbares en cas de résistance. ${ }^{5}$

1 Les recherches présentées ici sont une partie d'un projet dont les résultats sont publiés: voir SCHMIEDER: 2012, 2013, 2014a et 2017. La comparaison des sociétés esclavagistes et post-esclavagistes était un volet d'un projet mené par un groupe de chercheurs de l'Université d'Hanovre explorant les histoires post-esclavagistes de diverses sociétés de la Caraïbe et d'Afrique Occidentale.

2 Concept pour caractériser la croissance rapide de la production de la canne à sucre comme monoculture et la transformation de la société en une société esclavagiste de plantation (HIGMAN: 2000).

3 http://www.slavevoyages.org/assessment/estimates (29.09.2016).

4 Dans la Caraïbe, au temps des esclaves, une habitation désignait une plantation sucrière ou caféière. Dans cet article, je conserve en italique les termes utilisés dans l'historiographie sur cette époque et de cette région sauf mention contraire.

5 Le texte du Code Noir: http://www.axl.cefan.ulaval.ca/amsudant/guyanefr 1685.htm, (29.09.2016). Voir: «Article 33: L'esclave qui aura frappé son maître, sa maîtresse ou le mari 
L'abolition de l'esclavage par la Convention en 1794 ainsi que sa réintroduction en 1802 par Napoléon n'a pas concerné la Martinique occupée à cette époque par les Anglais (NICOLAS: 1996, pp. 280-285). La période entre 1789 et 1831 fut celle des révoltes des esclaves et des gens de couleur libres, notamment en 1789, 1800, 1811, 1822 et 1831 (GEGGUS: 1983 et 1996; THÉSÉE: 1993; TOMICH: 1990).

Après la révolution réussie des esclaves en Saint Domingue (Fick 1990, Cauna 1997, Popkin 2010), I'administration coloniale aurait voulu que la Martinique et la Guadeloupe supplantent Haïti dans la production de la canne à sucre. La production de sucre en Martinique était en augmentation, et pendant la période de l'esclavage atteignit son apogée en 1844, mais Cuba, île espagnole, gagna la guerre du sucre sur le marché mondial grâce à des innovations techniques favorisant une très forte productivité (TOMICH: 1990, pp. 30-31 et 76-111).

La traite transatlantique des esclaves ne fut abolie que sous la Monarchie de Juillet, en 1831 (JENNING: 2000, p. 32). Ceci entraîna quelques réformes en faveur des gens de couleur libres ${ }^{6}$ et procura une certaine «amélioration» de la condition d'esclave tout en donnant l'occasion aux frères de I'Instruction chrétienne de Ploërmel et aux sœurs de St. Joseph de Cluny de propager leur religion (DESLISLE: 1997, pp. 207-259; SCHMIEDER: 2013).

En 1847 il y avait environ 73.000 esclaves sur I'île (TOMICH: 1990, p. 87). Victor Schœlcher, abolitionniste et Sous-Sécretaire d'État à la Marine dans le Gouvernement de la deuxième République déclara I'abolition de

de sa maîtresse, ou leurs enfants avec contusion ou effusion de sang, ou au visage, sera puni de mort. Article 34: Et quant aux excès et voies de fait qui seront commis par les esclaves contre les personnes libres, voulons qu'ils soient sévèrement punis, même de mort, s'il y échet. Article 35: Les vols qualifiés, même ceux de chevaux, cavales, mulets, bœufs ou vaches, qui auront été faits par les esclaves ou par les affranchis, seront punis de peines afflictives, même de mort, si le cas le requiert. [...] Article 38: L'esclave fugitif qui aura été en fuite pendant un mois, à compter du jour que son maître l'aura dénoncé en justice, aura les oreilles coupées et sera marqué d'une fleur de lis une épaule; s'il récidive un autre mois pareillement du jour de la dénonciation, il aura le jarret coupé, et il sera marqué d'une fleur de lys sur l'autre épaule; et, la troisième fois, il sera puni de mort. [...]».

6 Centre des Archives d'Outre-Mer (CAOM), Aix-en-Provence, Fonds Ministériels (FM), Généralités (GEN), carton 160, dossiers 1320, Loi du 20.04.1833. Article 1: «Toute personne née libre, ou ayant acquis légalement la liberté, jouit, dans les colonies Françaises, $1^{\circ}$ del droit civile, $2^{\circ}$ del droit politique, sous les conditions prescrites par les lois.» 
l'esclavage par décret le 27 avril $1848^{7}$ (LARA: 2005, pp. 97-98). C'est deux ans plus tôt que débute notre histoire.

\section{LES SÉVICES AVANT L'ÉMANCIPATION}

Le point de départ en est le traitement d'un dossier de 1846 portant sur les sévices commis par Saint Cyrille Desgrottes, un planteur martiniquais, sur lequel l'Etat colonial a mené une enquête dans le contexte des réformes du système esclavagiste pendant la Monarchie de juillet (1830), notamment lors de la signature des lois Mackau de 1845, mais qui ne fut pas condamné.

Selon les lois Mackau, un maître pouvait être sanctionné s'il empêchait I'instruction religieuse de ses esclaves, les obligeait au travail le dimanche et pendant les fêtes religieuses, les privait de nourriture, de vêtements ou de soins médicaux, ou encore en cas de "traitement illégal", c'est-à-dire leur infligeait des punitions interdites; le tout classé sous la notion sévice» ${ }^{8}$.

Comme personne $n^{\prime}$ agit seulement en tant qu'individu, mais également en tant que membre d'une famille, $d$ 'une communauté et $d$ 'une classe (et dans le contexte colonial, d'une race, la catégorie la plus importante dans le

7 CAOM, FM, GEN, carton 153, dossier 1278, Extraits du Moniteur Universel (2., 3. , 4.,5.1848), Abolition de l'esclavage dans les colonies françaises. Décrets du Gouvernement provisoire et arrêtés du Ministre de la Marine et des Colonies, 27.4.1848.

8 CAOM, FM, GEN, carton 630, dossier 2736. Loi du 18 Juillet de 1845 relative au régime des esclaves dans les colonies (Loi Mackau), accompagnée de plusieurs ordonnances: Ordonnance du Roi qui fixe les formes à suivre pour faire concourir les fonds de l'État au rachat des esclaves, 26.10.1845. Ordonnance du Roi qui détermine la forme des actes relatifs au rachat des esclaves, etc., 23.10.1845. CAOM, FM, GEN, carton 139, dossier 1188. Etat des esclaves dans la distribution des fondes alloués par la loi du 19 Juillet 1845 pour l'achat, 15.8.1846. Archives des Sœurs de Saint Joseph de Cluny, Paris (ASJC), H 1 Ordonnances Royales relatives à l'esclavage, Ordonnance Royale concernant la nourriture et l'entretien des esclaves, 5.6.1846. Les années précédentes il y déjà eu plusieurs ordonnances sur le traitement des esclaves: CAOM, FM, GEN, carton 630, dossier 2736. Ordonnance du Roi concernant I'affranchissement des esclaves dans les colonies. 11.6.1839. Ordonnance du Roi relative à I'Instruction morale et religieuse des Esclaves dans les Colonies française ainsi qu'au Patronage que doivent exercer les Officiers du Ministère publique à l'égard de la même classe de la population 5.1.1840. CAOM, FM, GEN, carton 192, dossier 1476, Ordonnance du Roi relative à l'emprisonnement des Esclaves dans les Colonies françaises, 16.9.1841. Historiographie sur d'autres affaires de sévices: MOITT: 2001, pp.101-124; OUDIN-BASTIDE: 2008 et 2015. 
monde des idées de la Caraïbe française du XIXème siècle) ou encore d'un genre, je ne me concentrerai pas dans cet article seulement sur l'analyse du dossier concernant Saint Cyrille Desgrottes, mais considérerai également des documents trouvés dans les Archives d'Outre-Mer. D'autres membres de la famille Desgrottes y sont mentionnés dans les dernières années de l'esclavage, et pendant le processus l'émancipation de 1848 jusqu'au début des années 1870, lorsque la Troisième République légiférait sur les lois du travail dans le cadre du travail forcé. La famille Marraud des Grottes, désignée par «Desgrottes» dans les documents présentés ici, se composait dans les années 1840 des frères, André Vincent Saint Cyrille Desgrottes (1783-1874), Louis Marie Paul Desgrottes (1798-1877), Sainte-Marie Desgrottes (Vincent Joseph, 1782-1871) et Vincent Desgrottes (1793-1875). Leur mère, la veuve d'Isaïe Marraud des Grottes (1736-1813), Marthe Elisabeth Marie Carreau Gascherau avait vendu I'habitation à ses fils Saint Cyrille Vincent, Paul et Roseville (1795-1829). Les deux sœurs des frères Desgrottes encore vivantes (Elisabeth et Louise Félicité) n'apparaissent pas dans les sources que j'ai consultées. En 1828 Paul donc possédait l'habitation Marraud des Desgrottes en indivision avec ses frères Saint Cyrille et Sainte-Marie ${ }^{9}$ qui est parfois mentionnée sous la dénomination «habitation Veuve Desgrottes» (jusqu'en 1848), ou «habitation Paul».

Les faits : les sévices commis par Paul Desgrottes, Saint Cyrille Desgrottes et Prix Fortier, gérant de l'habitation de Sainte-Marie Desgrottes, sont énumérés dans une liste établie par le Ministère de la Marine et des Colonies le 8 janvier 1847. On peut aussi y lire: «S $S^{\dagger}$ Cyrille Desgrottes, aussi prop ${ }^{\text {re }}$ [propriétaire] au Macouba a été l'objet de deux poursuites successives. La première, pour châtiments excessifs et défaut de vivres, prescrite (sans suites, les faits de la plainte ayant été reconnus erronés); la seconde, relatée dans «la Réforme» [un journal libéral parisien] pour détention prolongée de

9 Pour la généalogie de la famille voir: http://bertrand.auschitzky.free.fr/AppendicesLabarre/ MarrauddesGrottes.htm (11.4.2016). II faut mentionner des contradictions avec d'autres sources consultées! Cette généalogie est intéressante dans la mesure où elle témoigne d'une endogamie stricte avec les autres familles békés de l'île. BRUNEAU-LATOUCHE, CORDIEZ ET CORDIEZ: 2002, pp. 813-816.

Au sujet de la plantation, cf.: http://www.culture.gouv.fr/public/mistral/mersri_fr?ACTION=CHERCHER\&FIELD_l=REF\&VALUE_l=IA97201068 (1 1.4.2016). http://bertrand.auschitzky. free.fr/AppendicesLabarre/macouba.htm (6.10.2016). 
deux de ses esclaves aux fers. Cette affaire était en cours d'instruction le 26 août $d^{\text {er. }}$ Le résultat ne nous est pas encore connu». ${ }^{10}$

L'enquête menée par les autorités coloniales sur le traitement des esclaves de cette famille a été rapportée dans «La Réforme» du 5 janvier 1847, sous le titre «Affaire v. Cyrille Desgrottes - M. Pujo». Cet article traite du cas de Saint Cyrille Desgrottes pour soutenir l'argumentation d'un certain $M$. Félice, auteur d'une pétition pour l'abolition immédiate et complète de l'esclavage qui dénonce l'oppression «plus ombrageuse et pesante» de planteurs sur leurs esclaves au moment où leur pouvoir est contesté. Effectivement, il dénonce que Saint Cyrille Desgrottes «irrité des modifications que les lois nouvelles ont apporté au régime disciplinaire, traita ses esclaves plus sévèrement que jamais et défendit particulièrement aux nourrices d'apporter leurs enfans [sic] au jardin» [NB : à l'encontre de l'usage dans les colonies qui permettaient aux mères $d^{\prime}$ 'allaiter leurs enfants sur le lieu de travail en cas de nécessité]. Le maître avait ordonné que les mères n'allaitent leurs bébés que le matin et pendant les pauses. Deux esclaves, Irène et Constance, ignorèrent l'ordre et se présentèrent le 8 août 1846 avec leurs nourrissons dans le jardin. Les deux femmes furent amenées immédiatement au galetas (petit grenier), une pièce de six mètres carré sans fenêtre qui servait de cachot dans cette plantation et n'était aérée qu'une fois par jour. «La Réforme» relate les propos $d$ 'Irène «Nous étions constamment dans l'ordure [...] nous mourions de chaud, de soif et de faim, ainsi que nos enfans [sic].» Les deux femmes firent un trou dans la toiture pour pouvoir respirer. Chargé de les punir, le père $d^{\prime}$ Irène, Elie, fut obligé de les mettre à la barre. ${ }^{11}$ Au bout de cinq jours de ce traitement, le mari de Constance, Cyprien, «s'échappa de l'habitation pour aller porter plainte au procureur du Roi de Saint-Pierre». Saint Cyrille Desgrottes sachant l'illégalité de cette détention, libéra les femmes. Cyprien quant à lui, fut reconduit à l'habitation par les autorités coloniales et ainsi exposé à la vengeance de son maître, bien que la gendarmerie détenait assez de preuves de l'emprisonnement illégal des

10 CAOM, FM, Série Géographique (SG), Martinique (MAR), carton 33, dossier 288, Esclavage - Sévices. Affaires de Sanois, Gosset, Desgrottes, Sully Vivier, 1847-1848. Liste des Sévices de la Famille Desgrottes (citation). Extrait des Greffes de la Cour Royale, 3.6.1846. La Réforme, 5.1.1847. Correspondance entre le Ministre d'Outre-Mer, le Gouverneur de la Martinique et le M. Pujo, Procureur du Roi du mars et avril de 1847. Saint Cyrille Desgrottes est mentionné dans ces dossiers sous le nom de Cyrille, $\mathrm{S}^{\dagger}$ Cyrille ou Saint Cyrille. La paroisse est celle de Sainte Anne de Macouba.

11 Une poutre munis d'espaces permettant de maintenir prisonnières les jambes d'un esclave pour le punir. 
femmes, «La Réforme» estimait qu'il n'y avait pas à attendre de sentence sévère contre Saint Cyrille Desgrottes vue «la criminelle entente entre des magistrats et des planteurs».

«La Réforme» cite également une lettre du Procureur du Roi, M. Pujo, au Maire de la commune de Macouba mentionnant les sévices infligés par Saint Cyrille Desgrottes aux esclaves, Eliséo et Sophie, ramenés à leur maître et attestant «l'administration (...) sage, douce et paternelle» de la plantation [italiques du journal]. Le journal évoque des cas similaires d'esclaves renvoyés à leurs maîtres après s'être plaints de sévices, et critique très fortement le comportement du Procureur du Roi en faveur des propriétaires et ainsi responsable de l'administration des sévices.

Ces documents montrent des formes de comportement social habituelles dans une société esclavagiste : un colon blanc chrétien ignorant les règles de l'humanité les plus simples, ${ }^{12}$ deux mères esclaves noires défendant leurs jeunes enfants contre un ordre cruel et arbitraire, un mari esclave noir défendant son épouse et son enfant, de nombreux esclaves qui protestent contre la maltraitance, mais aussi un père esclave obligé de mettre sa fille à la barre selon les ordres du maître. La justice coloniale défend les intérêts des planteurs contre les lois de la métropole.

Le courage d'Irène, de Constance et de Cyprien comme celui d'Eliséo et de Sophie, fournirent des arguments aux abolitionnistes blancs et noirs qui demandaient une abolition immédiate de l'esclavage du fait que cette institution non réformable devait être supprimée. Les révélations du journal suscitèrent une correspondance en mars-avril 1847 entre le Ministre de la Marine, le Gouverneur de la Martinique (l'Amiral Mathieu), le Procureur Général du Roi à la Martinique, $M$. Vaulx, et le Procureur du Roi à St. Pierre, M. Pujo. Une lettre de M. Pujo au Procureur Général du 6 avril 1847 détaille les sévices dénoncés et les plaintes déposées par les esclaves de Saint Cyrille Desgrottes, Sophie et Elysée (Eliséo est une erreur du journal). Sophie se plaignait du manquement de vivres et du travail de nuit pendant la roulaison de la canne et la fabrication du sucre. Elysée fut fouetté, incapable de dénombrer les coups qu'il reçut, et mis deux jours à la barre. $M$. Pujo accepta les justifications de Saint Cyrille Desgrottes argumentant que la morve était rare à cette époque de l'année et qu'il ne pouvait donner des portions complètes à Sophie, et affirmant qu'Elysée n'avait pas reçu

12 On peut légitimement se demander s'il était si cruel avec ses esclaves parce qu'il n'avait pas de fils légitimes dans sa famille pourtant nombreuse - son frère Paul avait treize enfants... http:// bertrand.auschitzky.free.fr/AppendicesLabarre/MarrauddesGrottes.htm (28.9.2016). 
plus des 29 coups de fouet permis. Après une visite à l'habitation de Saint Cyrille Desgrottes, M. Pujo affirma que les plaintes «[...] avaient le caractère de la malveillance, et, qu'il importait que dans un intérêt d'ordre, que ces deux esclaves fussent reconduits sur l'habitation de leur maître par la Gendarmerie."13

Ce dossier des Archives d'Outre-Mer concernant la famille Desgrottes contient également un extrait du procès de la Cour Royale fait à Louis Marie Paul Desgrottes, quarante sept ans, le 3 juin 1846, condamné à cent francs $\mathrm{d}^{\prime}$ 'amende et à payer les frais du procès parce qu'il "[...] a fait mettre des chaînes aux pieds de ses esclaves, Jérôme et Clet, et les a tenus enchaînés plus de quatorze mois, tout en continuant à les employer pendant les heures de travail pour les cultures et les condamnant à la barre de discipline de I'hôpital de son habitation la nuit et les jours fériés; $[. . .]^{\prime \prime} .^{14}$ Des accusations plus graves furent rejetées par la Cour. $M$. Pujo légitima les sévices octroyés par Paul Desgrottes pour refus de travail dans l'atelier de son habitation.

Sainte Marie Desgrottes et son gérant et «beau-frère» François Prix Fortier ${ }^{15}$ furent accusés de châtiments excessifs sur les esclaves Maxime, Dominique, Modeste, Zacharias et Césaire. Maxime fut mis huit jours à la barre et condamné aux quatre piquets ${ }^{16}$ parce qu'il "avait répondu avec insolence à quelques observations de l'économe, \& s'était oublié jusqu'à menacer de son couteau un esclave auquel ce dernier avait ordonné de l'arrêter". Les fers de 4,3 kilogrammes ne lui permettaient pas d'écarter les jambes. «Nonobstant de ces entraves, Maxime fut contrait lui aussi aux travaux de

13 CAOM, FM, SG, MAR, carton 33, dossier 288, Esclavage - Sévices. Affaires de Sanois, Gosset, Desgrottes, Sully Vivier, 1847-1848.

14 CAOM, FM, SG, MAR, carton 33, dossier 288. Extrait des Greffes de la Cour Royale, 3.6.1846. Ce document mentionne Louis Marie Paul Desgrottes qui apparaît dans l'ouvrage de LARA et BLANCHET: (2011) sous le nom de Paul Desgrottes accusé des sévices (p. 328). Cependant, dans cette liste, les personnes ne sont pas classés alphabétiquement ni par famille ou commune et les doubles mentions ne sont pas corrigées (p. 257: "Desgrottes, Sieur et Dame, elle née Fortier", c'est aussi Paul qui fut le mari d'une Fortier), pp. 278, 316, 318. II en résulte donc que l'on parle de la même personne que Louis Paul Desgrottes qui apparaît dans la liste des indemnités que j'ai vue (note 21). Pareil pour la page de généalogie où l'on parle de «Paul Marraud Desgrottes» (http://bertrand.auschitzky.free.fr/ AppendicesLabarre/ MarrauddesGrottes.htm) (5.5.2016); Paul Marraud Desgrottes est ainsi la même personne que Louis-Marie Paul Marraud Desgrottes, mentionné dans les autres sources.

15 Erreur du rapport, Fortier était le frère de Françoise Victoire Rose Fortier, 1802-1859, épouse de Paul Desgrottes. Sainte-Marie Desgrottes était marié avec Marie Marguerite Belloncle.

16 Séance de fouet pendant laquelle l'esclave était lié aux quatre membres et étendu à terre. 
la culture et attaché sans pitié à la barre de discipline les soirs et les jours de fête." Dominique, Modeste, Zacharias et Césaire furent punis pour "le même projet d'évasion qui avait provoqué le châtiment infligé à Jêrome \& à Clet par le S. Paul Desgrottes" et mis dans un cachot (les deux premiers pour un mois) trop étroit pour $y$ allonger complètement les jambes. Ils furent tous également condamnés aux quatre-piquets. Pendant 15 jours ils travaillèrent dans les champs enchaînés et mis à la barre lors des pauses, le soir et les jours de fête. Le gérant fut condamné à une amende de $200 \mathrm{~F}$ et aux frais du procès. ${ }^{17}$

Ces cas de résistance des esclaves cruellement punis démontrent une violente relation entre les frères Desgrottes et eux. Les Desgrottes avaient également proposé de faire expulser de la Martinique les "esclaves dangereux", Jean-Philippe et Cyprien (marrons au moment de la requête de Sainte-Marie Desgrottes) pour "complot d'évasion". ${ }^{18}$

\section{L'ÉMANCIPATION DE L'AN 1848}

L'esclavage à la Martinique fut abrogé le 23 mai 1848, alors que le Gouverneur Rostoland aurait dû le faire à la suite d'une insurrection des esclaves. Une déclaration du Gouvernement provisoire du 27 avril 1848, stipulait la fin de l'esclavage par proclamation du décret d'abolition deux mois après l'arrivée d'un Commissaire de la République dans chaque colonie esclavagiste. Le commissaire chargé de la Martinique, Perrinon, arriva le 4 juin, ce qui impliquait la fin de l'esclavage au plus tôt le 4 août. Les esclaves qui doutaient de la proclamation de leur liberté (annoncée le 5 avril dans le Journal Officiel de la Martinique), s'étaient insurgés le 22 mai, touchant particulièrement St. Pierre, mais aussi «les trois habitations Desgrottes au Macouba" qui "ont été (...) brûlées ou saccagées". ${ }^{19}$ La famille Desgrottes fut ciblée par la vengeance des esclaves maltraités mais personne n'a été blessé, seuls les biens furent endommagés. L'abolition de l'esclavage $\mathrm{n}^{\prime}$ a pas permis l'indemnisation des victimes mais celle de ses profiteurs. Desgrottes, Joseph Ste-Marie; Desgrottes, Louis Paul Marie;

17 CAOM, FM, SG, MAR, carton 33, dossier 285, cité de la lettre du Procureur Général au Gouverneur, 8.6.1845. Lettre du Gouverneur au Ministre 9.6.1845, Extraits des minutes des greffes de la Cour Royale de l'île de la Martinique, 4.6.1845.

18 CAOM, FM, SG, MAR, carton 42, dossier 350. Esclaves dangereux. Expulsions 1838-1847. Rapport en Conseil, 13.1.1845. Arrêté du Gouverneur 4.2.1845.

19 CAOM, FM, SG, MAR, carton 11, dossier 108, Lettre imprimée du planteur Pécoul au Chef du journal National sur les événements du 22 et 23 mai 1848. 
qui signa également du nom de son épouse Rose Victoire Desgrottes, née Fortier, Desgrottes, St. Cyrille André Vincent, mentionnés dans cet article; Desgrottes, Jean-Joseph Marie et Desgrottes, Pierre Joseph Aristide, la société P. Desgrottes \& Cie., Marraud Desgrottes, Pierre Etienne Elisabeth Roseville; $D^{m e}$ Ve Marraud Desgrottes, Henri Just, née Lagrange (Marie Joseph Jeanne Robert); $D^{m e} V^{e}$ Marraud Desgrottes, Jean Baptiste Isaïe, née Carreau Gascherau (Marthe Elisabeth, la mère des propriétaires d'esclaves mentionnés dans le cadre des sévices dénoncés), ${ }^{20}$ et Marraud Desgrottes, Marie Henry Constant, furent indemnisés de la perte de leurs esclaves. La somme individuelle la plus importante fut octroyée à Sainte Marie Desgrottes $(11.479,44 \mathrm{~F})$. La totalité des membres de la famille mentionnée ici reçut la somme de 42.595,35 F sur plus de trente millions de Francs d'indemnité coloniale prévue pour l'île. ${ }^{21}$

Dans les archives on trouve toujours des traces de l'élite sociale, ce qui n'est pas forcément le cas pour les subalternes, notamment les esclaves, difficiles à retrouver lorsqu'ils ont changé de nom à leur libération voire impossibles à retrouver s'ils ont quitté leur commune $d^{\prime}$ inscription. ${ }^{22}$ Les actes d'individualité ne mentionnent ni les anciens maîtres, ni les habitations où les anciens esclaves ont vécu et travaillé. On ne peut les retrouver que dans les livres des naissances de la population esclave (où apparaît également le nom du maître et l'habitation) mais accessibles qu'à partir de 1834 sur la commune de Macouba. Ainsi, je n'ai pu identifier ni Sophie ni Elysée, mais retrouvé les bébés victimes de Saint Cyrille Desgrottes ainsi que leurs mères.

20 La généalogie (geneanet.com) note sa naissance en 1761. Comme je n'ai trouvé aucune mention de sa mort dans aucune source sous cette combinaison de noms, elle aurait donc eu 87 ans au moment de l'émancipation.

21 CAOM, FM // K 14 Indemnité coloniale. Colonie de la Martinique. État des règlements définitifs. $N^{\circ} 2$ (Exposé sommaire alphabétique des indemnisés, qui mentionne aussi des membres des branches familiales Desgrottes Desmadrelles, Desgrottes de S' Just et l'indemnité que recevait la veuve de François Prix Fortier, Jeanne Rose Victoire, née Assier de Montrose. II n'est pas possible d'identifier clairement tous les membres de la famille Desgrottes sur la liste sans plus d'informations du fait de la répétition des prénoms, il y a par exemple plusieurs Aristide, Henri, Just, Pierre etc. LARA: 2005, p. 961. 30.197.174,20 F d'indemnité coloniale furent allouées aux propriétaires martiniquais sur la base de $409.98 \mathrm{~F} /$ par esclave.

$22 \mathrm{Si}$ l'on ne connaît pas le nom choisi par un nouvel affranchi après l'émancipation, on ne le trouve pas dans la base de données de "anchoukaj.org» (une base des dates du Comité du Marche 23 Mai 1998 pour aider aux descendants des esclaves à trouver leurs ancêtres). 
La fille d'Irène, au cachot avec sa mère, a survécu aux sévices: Adelaïde, "enfant noir", fille d'Irène, 28 ans à l'époque, était née esclave de Saint $\mathrm{Cy}$ rille Desgrottes le 8 septembre 1845. Inscrite au registre d'état civil le 5 février 1849 sous le nom d'Adelaïde Afonne, elle fut légitimée en 1853, comme son frère Louisy Afonne, par le mariage de sa mère Irène Afonne avec Toussaint Grain. II n'y pas de notice concernant la légitimation d'une autre sœur de un an, Louisonne, peut-être déjà décédée au moment du mariage. Lors de leur mariage, Toussaint Grain, nouveau libre et cultivateur, avait 42 ans et Irène Afonne 36. Ils reconnurent en même temps deux enfants nés depuis l'émancipation, Juliette Afonne, deux ans, et Thomas Afonne, un an. ${ }^{23}$

La fille de Cyprien et Constance, Pascaline, également enfermée au cachot avec sa mère, est née esclave de Saint Cyrille Desgrottes le 5 mai $1846 .{ }^{24}$ La crainte de sa mère, non autorisée à nourrir son enfant pendant le travail, était peut-être dû à la perte de Faustin à deux ans le 3 janvier $1844,{ }^{25}$ un autre de ses enfants. Pascaline fut inscrite au registre des individualités en février 1849 sous le nom de Pascaline Chatelot. Toute la famille fut alors déclarée, Cyprien Chatelot, 43 ans, né dans la commune de Macouba, fils d'Angélique décédée, sa femme Constance Chaly lépouse légitime malgré son nom de famille différent), 39 ans, née dans la commune de Macouba, fille de Charlotte décédée, et leurs enfants, Charlotte Chatelot, 12 ans, et Noëlise Chatelot, 9 ans. Les sœurs de Cyprien furent également déclarées. II s'agit de Mélanie Chatelot (48 ans) avec ses enfants, Claire Chatelot (27 ans), Louise Chatelot (23 ans), Rosalie Chatelot (16 ans) et Dieudonné Chatelot (13 ans) et ses petites-filles: Marie Clairine (4 ans) et Agnès (2 ans), les filles de Claire, ainsi que Roseline (1 an), fille de Louise; ensuite, $d$ 'Emmérancienne Chatelot (35 ans) avec ses enfants Marie Désirée

23 Archives départementalesdelaMartinique(ADM): http://www.patrimoines-martinique.org/?form_ search_fulltext $=\% 5 \mathrm{~B} \% 3 \mathrm{Asubject} \% 5 \mathrm{DEtat}+$ civil $+\mathrm{ET}+\mathrm{int}+\mathrm{ET}+\% 5 \mathrm{~B} \% 3 \mathrm{Ageogname} \% 5 \mathrm{DMacou}-$ ba\%2OSAUF+\%5B\%3Aunitid\%5D26J * \&btn_valid=\%C2\%A0\%C2\%A0\%C2\%A0\%C2\%AOTrouver\&action=search\&id=chercher_liste (6.10.2016). État civil. Population esclave: naissances, mariages, décès (commune du Nord), Macouba, 1845, Cote: Edepot16/E28, n 115 (naissance de Adelaïde). Registre d'individualité, Macouba, Nouveaux-libres, 1849, Cote: Edepot16/ E32, $N^{\circ}$ 485-489. CAOM-IREL, http://anom.archivesnationales.culture.gouv.fr/caomec2/ (24.10.2016). Inventaires détaillés, État civil, Macouba 1853 (tous actes), № 26, 7.2.1853 (mariage $d^{\prime}$ Irène Afonne et Toussaint Grain).

24 ADM, voir note antérieure: État civil. Population esclave: naissances, mariages, décès, Macouba, 1846, Cote: Edepot16/E29, No37.

25 Ibidem, Macouba, 1844, Cote: Edepot16/E26. 
(5 ans) et Martin ( 2 ans); puis Segonde Chatelot (38 ans) avec ses enfants Céleste (15 ans), Rupert (13), Sully (8 ans), Reine (6 ans) et Solitude (2 ans). Ces derniers furent légitimés par le mariage de leur mère avec Gaspar Paille en 1851. A ce moment Gaspar Paille, nouveau libre et cultivateur, avait 50 ans et Segonde Chatelot 40 . Ils vivaient tous les deux sur I'habitation Chénaux à Macouba. ${ }^{26}$ Ils purent reconnaître leurs six enfants ainsi que la petite Marie Angélique, deux ans, née après l'émancipation et une fille ainée, Anna (21 ans), non enregistrée avec ses parents dans les actes $\mathrm{d}^{\prime}$ individualité. ${ }^{27}$ II est important de signaler que nous avons pu retrouver une famille complète ayant résisté à l'esclavage et survécu. Ainsi, la discussion qui porte sur le fait de savoir si les esclaves vivaient dans des familles nucléaires ou matrifocales, a peu de sens; il existait sans doute les deux modèles et ce, même au sein d'une famille. ${ }^{28}$ On peut également finalement conclure que des pères d'enfants esclaves reconnaissaient bel et bien leurs enfants et en prenaient la responsabilité. L'absence générale des pères des enfants esclaves est un mythe. ${ }^{29}$

26 En 1852 Sainte Marie Desgrottes avait vendu une partie de l'habitation à Gustave Chéneaux. http://bertrand.auschitzky.free.fr/AppendicesLabarre/macouba.htm (6.10.2016).

27 ADM, voir note 18, État civil, Registre d'individualité, Macouba, Nouveaux-libres, 1849, Cote: Edepot16/E32, No 380-395, 409-412, 429-430. CAOM-IREL, voir note 18, Inventaires détaillés, État civil, Macouba 1851 (tous actes), No. 125 (Mariage de Segonde Chatelot et Gaspar Paille).

Dans le registre d'Etat civil de Macouba (CAOM-IREL) 1849-1850 est inscrit : 1849, № 10 , 1.2.1849: Une fille de Segonde Chatelot, Marie Angélique, née le 15 janvier 1849. № 61 , 27.4.1849: Roseline Chatelot, fille de Louise Chatelot, morte le 26 d'avril 1849 sur l'habitation de Paul Desgrottes. N ${ }^{\circ}$. 128, 30.8.1849: Une fille de Cyprien Chatelot et Constance, Olympe, née le 8 août 1849 sur l'habitation Chénaux. № 115, 27.7.1850: Une fille d'Émérancienne Chatelot, Févrilette, née le 1 juillet 1850 sur I'habitation Chéneaux.

28 Durant ces dernières décennies, il y eut de nombreuses discussions pour savoir si les esclaves aux Amériques et particulièrement dans la Caraïbe pouvaient fonder des familles et si oui, quelles formes elles avaient. L'idée qu'il n'y avait pas de famille nucléaire, que les enfants ne connaissaient pas leurs pères ou que son absence ainsi que la matrifocalité durant la période de l'esclavage ont engendré des familles centrées sur la mère et les fuites des hommes de l'espace domestique tout au long du XXe siècle jusqu'à aujourd'hui, est fortement contestée. Durant les dernières décennies de l'esclavage, sur des îles françaises et anglaises, il existait des plantations où les esclaves vivaient en familles selon le modèle «couples avec enfants», ce qui était impossible sur les grandes plantations à l'ouest de Cuba où les hommes africains étaient beaucoup plus nombreux que les femmes. Sur le débat cf.: POTTHAST: 1998, SCHMIEDER: 2017, pp. 43-46 et 62-72. Sur Cuba, cf.: SCHMIEDER: 2014b, pp. 233-234.

29 Pour plus de détails sur les anciens esclaves et leurs rôles en tant que pères et mères: SCHMIEDER: 2014a, pp. 21-35. 
Depuis l'émancipation - malgré les craintes d'une grande vengeance à la manière haïtienne - les nouveaux affranchis n'ont tué aucun Blanc et préféraient célébrer leur nouvelle liberté (PAGO: 2006; SCHMIEDER: 2017, pp. 151-154). Et ce, même si dans les mois suivants, ils ont pillé, incendié et détruit les plantations des békés les plus détestés pour les sévices envers leurs esclaves, parmi eux, les Sieurs Desgrottes. [NB : dans la commune $S^{\text {te }}$ Anne de Macouba]. «L'habitation Ve Desgrottes est la seule où il ait eu dévastation et vol dans le temps dernier. Trois expulsions ont suffi pour y rétablir l'ordre" ${ }^{30}$

Le Commissaire de la République Perrinon chargé d'organiser le nouveau système du travail par "contrats d'associations" (contrats de travail entre propriétaire et communauté des nouveaux libres/cultivateurs) déclara en juillet 1848 au Ministère que les problèmes rencontrés pour trouver un accord de travail dans les habitations Fortier, Pompignan et Desgrottes, dans la commune de Basse Pointe, résultaient de «l'attitude gardée par ces citoyens envers les cultivateurs" qui n'étaient pas prêts à cesser de les traiter en esclaves. À Macouba: "Sur l'habitation Paul Desgrottes les cultivateurs ne demandaient qu'à s'arranger et à prendre le travail mais le citoyen Desgrottes a persisté à vouloir les renvoyer sous prétexte qu'il avait trop de bras relativement à l'étendu de ses terres. Les cultivateurs, de leur côté, déclaraient ce motif inexact, et déclaraient qu'un appel avait été fait à des cultivateurs étrangers; il m'a fallu les prendre par leur amour propre et leur dire que des hommes libres ne pouvaient rester chez autrui contre son gré, pour les décider à chercher de l'emploi autre part. ${ }^{\prime 1}{ }^{11}$ Le fait que Paul Desgrottes remplace ses anciens esclaves par d'autres travailleurs leur faisait perdre leurs cases sur l'habitation et les jardins y attenants.

Lors d'une inspection sur I'habitation Paul et Sainte Marie Desgrottes en août 1848, on lit qu'il n'y a «Pas encore de condition, le Citoyen Desgrottes refusant $d^{\prime}$ 'envoyer de $\mathrm{S}^{\dagger}$. Pierre, où il se trouve, des mandataires pour stipuler en son nom" ${ }^{32}$ Craignaient-ils une vengeance de leurs anciens esclaves? En tout cas, l'attitude hostile des frères Desgrottes envers les cultivateurs

30 CAOM, FM, SG, AMER (Amérique), 2400 COL 93, 2, Rappel des émeutes de 1848 et 1849. Note sur les vols, pillages, incendie, excès de Mai 1848, à la Martinique, et de juin 1849. 14.2.1850. CAOM, FM, SG, MAR carton 56, dossier 464, Lettre de Perrinon au Ministre de la Marine et Colonies, 9.10.1848 (citation).

31 CAOM, FM, SG, MAR, carton 56, dossier 464, Rapport de Perrinon du 25.7.1848.

32 CAOM, FM, SG, MAR, carton 56, dossier 464, Etat indicatif des tournées opérées par les commissaires du travail dans les diverses Communes de la Martinique, sans date. Du contexte on peut supposer que le document fut écrit en août de 1848 . 
dans les mois qui suivirent l'émancipation a peut-être aussi eu une influence sur la destruction partielle de la plantation en octobre de cette même année.

\section{Les CONFLITS DE LA PÉRIODE POST-ESCLAVAGISTE}

Le travail organisé comme il l'était en Martinique après l'esclavage était de facto du travail forcé obtenu par des décrets contre «le vagabondage et la mendicité». Le Gouvernement de la République avait ordonné l'expulsion des travailleurs des cases installées sur les plantations si les anciens esclaves/nouveaux libres ne travaillaient pas selon les conditions stipulées dans les contrats d'associations. Ceux qui résistaient contre le travail forcé se retrouvaient rapidement dans les "ateliers de discipline" (les décrets: LARA: 2005, pp. 105-108; la pratique: TOMICH: 1995; SCHMIEDER: 2017, pp. 239-251). Les jurys cantonaux, des médiateurs entre employeurs et employés, fonctionnaient plutôt comme un instrument des propriétaires et de I'État colonial contre les travailleurs (COTTIAS: 2004; SCHMIEDER: 2017, pp. 246-248). ${ }^{33}$ La situation s'aggrava encore sous I'Empire. A partir de 1852 le code des lois du travail contenait des articles "contre le vagabondage", prévoyait un impôt personnel pour les nouveaux affranchis, impliquait l'obligation de posséder un livret de travail, où étaient inscrits les contrats de travail et les dettes contractées, l'exclusion des travailleurs et de leurs enfants du système éducatif due à une taxe prohibitive pour droit $d$ 'inscription aux écoles publiques. Ainsi les nouveaux libres se voyaient obligés de travailler dans les champs conformément aux conditions des propriétaires (RENARD: 1993; SCHMIEDER: 2017, pp. 258-259 et 339-342; SCHMIEDER: 2013, pp. 167-168). Les salaires furent revus à la baisse par l'arrivée de travailleurs contractés d'Afrique, d'Inde et de Chine, une immigration forcée (MASSÉ: 1980; CARDIN: 1990; SMERALDA-AMON: 1996; NORTHRUP: 2000). Un des frères Desgrottes s'était prononcé en faveur de cette nouvelle immigration forcée de travailleurs aux "[des] habitudes laborieuses". II interprétait comme des "tendances des indigènes à $s^{\prime}$ affranchir du travail régulier et soutenu", ${ }^{34}$ le désir des populations descen-

33 Les essais de travailleurs pour gagner les jurys à leur cause ont tourné court, également pour ceux de la famille Desgrottes. CAOM, FM, SG, MAR, carton 164, dossier 1499 Jurys cantonaux, Etat A Jurys cantonaux, $3^{\text {ème }}$ trimestre 1849, Matière Civile, Arrondissement Fort-deFrance, Canton Marin, 22.9.1849, Argan, cultivateur demeurant à Ste. Anne contre Desgrottes, propriétaire demeurant à $S^{\text {te. }}$ Anne, "Réclamation du montant du travail fait sur une propriété", le résultat: "Le demandeur débouté et condamné aux dépens."

34 CAOM, FM, SG, MAR carton 129, dossier 1136, Conseil privé 274.1.1852, Audience des planteurs Le Pelletier Duclary, Wallée Clerc, Northum de Percin, Bally, Desgrottes sur l'immigration asiatique. 
dant $d^{\prime}$ anciens esclaves qui ne voulaient plus travailler pour leurs anciens maîtres pour de faibles salaires et plutôt être des agriculteurs indépendants, produisant pour leurs propres besoins et les marchés locaux.

A cette époque du travail forcé des descendants d'esclaves et des $\ln$ diens, il y eut beaucoup d'incendies volontaires et de protestations, notamment dans les plantations de la famille Desgrottes. La résistance des cultivateurs frappa certains fonctionnaires de l'État obligés d'appliquer des lois répressives. Ainsi, Adolphe Julien, commis à la police de la commune de Macouba, fut traité de "cochon" et de "canaille" par un travailleur anglais, David (donc un immigré des îles anglaises de la Caraïbe) sur l'habitation Desgrottes à Macouba qui fut condamné à six mois d'emprisonnement, à 100 francs $d$ 'amende et à payer les frais du procès (69 francs)..$^{35}$ Alors qu'un travailleur gagnait 1 Franc par jour, en comparaison avec les faibles punitions pour sévices, nous en déduisons le caractère raciste, voire de classe, de la justice coloniale.

Sur l'habitation Desgrottes, les Indiens immigrés n'étaient pas plus satisfaits de leur sort que les nouveaux libres et leurs enfants. Quelques Indiens incendièrent une case en bagasse sur l'habitation Desgrottes à Sainte Anne (Macouba) dans la nuit du 27 au 28 septembre 1859. Quelques jours plus tôt, le matin du 23 août, Moutou, Coupa-Moutou, lequel avait signifié son mécontentement à l'administration de l'habitation Desgrottes, et Cojandé avaient été enfermés pour avoir couvert l'économe de l'habitation d'excréments humains. Le lendemain, les trois Indiens, ainsi que sept autres, s'enfuirent se réfugier au Commissariat de l'immigration. Le commissaire les renvoya à l'habitation Desgrottes où ils furent enfermés et punis. L'incendie qui s'en suivit fut donc un acte de vengeance. Les responsables ne furent jamais arrêtés, même si Coupa-Moutou et Cojandé furent fortement suspectés. Ces Indiens avait également planifié une évasion vers $\mathrm{S}^{\text {te }}$ Lucie, I'île anglaise voisine, gouvernée par le créole Martin, ${ }^{36}$ qui n'aboutit pas.

La colère et le désespoir des travailleurs agricoles afro-martiniquais, descendants d'esclaves, et des immigrés indiens soumis au travail forcé, à la pauvreté, au racisme et à l'injustice des tribunaux coloniaux s'exacerba lors de "l'insurrection du Sud" du 22 au 26 septembre 1870 (à Rivière-Pilote, puis à Vauclin, Sainte Anne, Le Marin, Rivière-Salée et Saint Esprit), déclenchée par un ravitaillement mal assuré pour cause de guerre franco-al-

35 CAOM, *6 Dépôt des Papiers Public (DPPC), MAR, tome 876, 1853-54, Cour d'Appel, $n^{\circ} 27$, 10.4.1854.

36 CAOM, FM, SG, MAR, carton 12, dossier 119, Lettre du Procureur Général au Gouverneur du 10.10.1859 sur une série $d$ 'incendies dans les plantations. 
lemande et de proclamation de la Troisième République (vue globale de la rébellion: PAGO: 2011). L'affaire "Lubin"37 est la première liée à cette insurrection. Léopold Lubin, a été condamné à cinq ans de détention au bagne de Guyane et à une amende de 1.500 Francs pour coups et blessures contre un Blanc, le commissaire adjoint Augier de Maintenon, alors que ce dernier $n^{\prime}$ a pas été inquiété pour les mêmes faits envers Lubin.

Louis Cléo Codé, un colon, fut tué pendant l'insurrection. II avait été membre du jury qui avait condamné Lubin, voté cette dure sentence et hissé le drapeau blanc sur son habitation par mécontentement contre l'avènement de la Ille République. II reproduisait ainsi le geste des ultra-royalistes qui avaient hissé le drapeau blanc lors de la «révolte de Grande Anse» des abolitionnistes noirs martiniquais en 1833, montrant son adhésion à I'ancien régime et à l'esclavage (HARTKOPF SCHLOSS: 2009 : 155-156).

A cette époque, un autre évènement provoqua la colère des travailleurs de la famille Desgrottes. Eugène Desgrottes (né en 1837, fils de Paul Desgrottes dont nous avons déjà parlé et cousin d'Henri Desgrottes dont nous parlerons plus tard) a été condamné en 1868 à payer une amende de 50 Francs et les frais du procès (un jugement de la Cour l'avait acquitté en première instance mais fut rejeté par le Procureur Général) pour avoir battu un travailleur immigré indien avec un bâton. Eugène Desgrottes avait justifié ces coups pour violence envers le chef $\mathrm{d}^{\prime}$ atelier, ${ }^{38}$ condamnation particulièrement légère comparée à celle de Léopold Lubin condamné pour des faits similaires. Dans une correspondance, le Gouverneur Cloué écrivit à propos de la situation générale du traitement des travailleurs noirs ou d'origine indienne sur l'île avant et après 1870: «[...] on ne peut pas nier que quelques coups de houssine soient appliqués par-ci par-là. Le délinquant est puni comme l'a été $M$. Desgrottes, quand le fait est connu, mais je suppose qu'il est beaucoup de ces délits dont le bruit ne parvient pas jusqu'au chef-lieu." ${ }^{\prime 39}$

37 CAOM, DPPC, Greffes, Cour d'Assises, Fort-de-France, tome 915, 19.08.1870, Procédure criminelle contre Léopold Lubin. CAOM, FM, SG, MAR, carton 168, dossiers 1529, 1531, documents sur la grâce en faveur de Lubin, ordonné par le Gouverneur en novembre de 1873.

38 CAOM, FM, SG, MAR, carton 152, dossier 1367. Lettre du Procureur Général La Rougery au Gouverneur, 27.1.1873, en réaction aux rapports de $M$. Schœlcher dans le Journal d'OutreMer du 22.10.1872 sur la continuation des sévices dans les colonies caraïbes. Le Procureur Général ajoutait qu'il y avait eu entre le 1.9. et le 1.12.1872 seize poursuites et condamnations des propriétaires par mauvais traitements envers leurs ouvriers. Les condamnations consistaient à payer une amende entre 250 et 50 Francs.

39 CAOM, FM, SG, MAR, carton 152, dossier 1367. Lettre du Gouverneur au Ministre de la Marine et des Colonies. 8.2.1873, sur le problème décrit dans la note antérieure. 
Les émeutes de 1870 n'ont pas non plus épargné l'habitation d'Henri Desgrottes où la case en bagasse et les écuries furent incendiés, des moutons volés et la maison du maître pillée. ${ }^{40}$ Le Gouverneur informa le Ministre de la réaction d'Henri Desgrottes (né en 1815, fils de Louis Jean-Baptiste Marraud des Grottes (1785-1834) et de Joséphine Marie Dublanc Laborde, neveu de Paul, Saint Cyrille et Sainte Marie Desgrottes) sur les événements survenus sur leur plantation en 1870 : "M. Henri Desgrottes, un chef de ce parti réactionnaire, ami du rédacteur en chef des Antilles, ayant, depuis que l'insurrection a été étouffée, refusé de donner à ses immigrants plus de la moitié de ce qu'il leur doit de nourriture y ayant attaché à un arbre un africain pour le souffleter, j'ai donné l'ordre au Directeur de l'Intérieur d'assurer partout l'exécution des engagements contractés vis-à-vis des travailleurs. M. Desgrottes est venu me voir pour protester contre cette mesure, $s^{\prime} i l$ ne nourrissait pas ses indiens [sic], m'a-t-il dit, c'est par ce qu'ils avaient profité de l'insurrection pour le piller." Le Gouverneur avait signifié à Monsieur Desgrottes, que les Tribunaux, et non les propriétaires, devaient punir les coupables de la rébellion. ${ }^{41}$

Ainsi, les histoires liées aux sévices envers les travailleurs esclaves et plus tard «libres" tout comme les actes de résistance ne s'arrêtent pas brusquement en 1848. Les maîtres d'esclaves restaient les propriétaires et les nouveaux libres restaient les exploités et les conflits du temps de l'esclavage ont perduré, imprégné les mémoires et influencé les comportements des parties prenantes du conflit pendant des décennies.

\section{UN HÉRITAGE ENCORE VIVACE AUJOURD'HUI? ${ }^{42}$}

Alors que la Martinique est aujourd'hui un département français d'OutreMer et fait ainsi partie de l'Europe, j'ai été choquée lors de mon voyage en 2006 par la pauvreté ambiante et les infrastructures déficientes qui évoquent encore ce passé colonial.

40 CAOM, FM, SG, MAR, carton 12, dossier 121, annexe à la «Pétition pour obtenir la grâce des chefs révoltés condamnés à mort par l'opinion d'une partie de la population» $d^{\prime}$ Emmanuel de Lagrange et de Lavernais, 1.10.1870, liste des habitations pillées et incendiés, p. 5.

41 CAOM, FM, SG, MAR, carton 12, dossier 121. Rapport confidentiel du Gouverneur du Loisne au Ministre de la Marine et des colonies, 1.1 1.1870. Des cultivateurs indiens et africains introduits après l'émancipation des esclaves travaillaient sur la plantation Desgrottes.

42 Dans le cadre de cet article qui présente les résultats d'une recherche sur l'esclavage et la période immédiate post-esclavagiste, je n'ai pas pu me rendre à Macouba constater si des descendants des familles Chatelot et Afonne y vivent encore. 
Ceci n'est pas seulement une impression personnelle. Les taux de chômage et de pauvreté ainsi que les prix sont plus élevés qu'en France alors que le niveau d'éducation est plus bas, ${ }^{43}$ ce qui a provoqué des manifestations en Martinique et en Guadeloupe en 2009 (SMÉRALDA: 2009).

Estimant ne pas avoir de perspective en Martinique, beaucoup quittent I'île pour se rendre en France (GIRAUD et al.: 2009). Cette situation peut s'expliquer par le fait qu'il n'y a jamais eu de réforme agraire pour une redistribution des terres mais aussi parce contrairement aux victimes de l'esclavage, les propriétaires ont été indemnisés pour la libération des esclaves; ceci aggrava le clivage social entre les békés et les descendants d'esclaves. Par ailleurs, la monoculture, aujourd'hui la banane a remplacé la canne à sucre mis à part quelques distilleries rhumières, produit une forte dépendance aux prix du marché mondial.

Aujourd'hui il est possible de visiter l'ancienne habitation de la famille Marraud des Grottes, "l'habitation Paul" où l'on produit de la banane. ${ }^{44}$ Le guide touristique du site web ne mentionne pas son passé esclavagiste. Le silence ou la minimisation de ce passé est classique pour la présentation touristique des plantations comme sites historiques (CHIVALLON: 2006). La reconnaissance de la traite et de l'esclavage comme crimes contre l'humanité (Loi Taubira 2001) et du 10 Mai comme date de la «journée nationale des mémoires de la traite, de l'esclavage et de leur abolition» (2006), ${ }^{45}$ montrent que la politique commence à changer. Les institutions publiques de la Martinique mentionnent aujourd'hui plus souvent les héritages négatifs de l'esclavage et privilégient leurs présentations de ce passé dans le Domaine-Musée de La Pagerie ${ }^{46}$ comme le montre notamment l'évocation du rôle de Joséphine Beauharnais, originaire de la famille de planteurs martiniquais Tascher de la Pagerie et plus tard impératrice, dans le rétablissement de l'esclavage, qui avait été aboli par la Convention en 1794, par Napoléon Bonaparte, Premier Consul en 1802.

43 En Martinique le chômage concerne 26 à $30 \%$ de la population contre $9 \%$ en France, le taux de Martiniquais sans diplôme est de $38 \%$, celui de la pauvreté des ménages de $17 \%$ contre $7 \%$ en France et $20 \%$ des actifs reçoivent le RMI (revenu minimum d'insertion) contre $3 \%$ en France (PAQUET: 2009, pp. 89-90).

44 http://www.culture.gouv.fr/public/mistral/mersri_fr?ACTION=CHERCHER\&FIELD_1 = REF\&VALUE_l $1=\mid$ A997201068 (11.4.2016).

45 Il est impossible de citer ici tous les ouvrages sur les mémoires de l'esclavage en France et aux Antilles françaises; je cite cependant : COTTIAS: 2005; REINHARDT: 2006; MICHEL: 2015; FRITH et HODGSON: 2015.

$46 \mathrm{http}: / /$ www.esclavage-memoire.com/evenements/josephine-l-esclavage-domaine-de-la-pagerie-les-trois-ilets-martinique-2 17.html (25.10.2016). 
Ces comportements ne touchent pas I'héritage des familles békés vivant encore là-bas. La famille Marraud des Grottes conserve ses propriétés, joue un rôle important dans l'économie bananière de la Martinique et perçoit des subventions de l'Union européenne. ${ }^{47}$ Elle n'est qu'un exemple alors que d'autres Békés, dont les noms apparaissent sur les listes des sévices, perçoivent aussi des subventions. ${ }^{48}$ Je n'ai pu vérifier si des membres de la famille Marraud des Grottes ont signé la déclaration «Nous nous souvenons» lancée en 1998 à l'occasion du Cent-cinquantenaire de l'Abolition de l'esclavage par Robert de Jaham sur le site web de l'organisation «Tous créoles !» par laquelle des descendants des colons reconnaissent leur rôle dans l'esclavage. Ce court texte cependant, cache plus qu'il ne le révèle le passé esclavagiste, ne s'excuse pas auprès des descendants des victimes et escamote les conséquences de l'esclavage sur la longue durée. ${ }^{49}$

47 http://outremers360.com/economie/la-banane-la-revolution-environnementale/(28.9.2016), interview de Jean-Claude Marraud des Grottes le 18.5.2016, responsable du projet «Banane française» comme stratégie marketing. (Jean-Claude Marraud des Grottes, né en 1927, neuvième génération de la famille, voir http://bertrand.auschitzky.free.fr/AppendicesLabarre/MarrauddesGrottes.htm (28.9.2016). Nicolas et Marie-Jose Marraud des Grottes (voir paragraphe suivant) y apparaissent en huitième génération. Nicolas Marraud des Grottes est Président du groupement des plus gros producteurs de bananes «Banamart».

https://blogs.mediapart.fr/lisadavid/blog/030213/tour-de-martinique-des-subventions-europeennes (28.9.2016): Sur les békés et les subventions perçues par l'Union Européenne : «La 'Sarl Macouba' de Marie-Joseh Marraud Des Grottes obtient en 2010 au titre du Feaga et du Feader la somme de $1270481,06 €$ et en 2011 celle de $1317774,64 €$.» «'Perinelle' de JeanClaude Marraud Des Grottes reste agri-millionnaire avec une subvention de 1 171 252,48€ en 2010 et un peu plus de 1193 221,19€ en 2011.» «Les aides européennes de 'Socoban' de Nicolas Marraud Des Grottes se situent au même niveau avec 329 152,68€ en 2011 .»

48 Par exemple Huyghes Despointes, Simonet et Jaham : https://blogs.mediapart.fr/lisadavid/ blog/030213/tour-de-martinique-des-subventions-europeennes (28.9.2016), CAOM, FM SG MAR, carton 169, dossier 1538. Comptes rendues d'assises. Jugements de sévices contre les esclaves. 1847-1848, Report du Procureur Général au Gouverneur, 26.3.1848: Louis Noël Huyghes Despointes fut condamné à 2 mois d'emprisonnement, à une amende de 200 Francs et à payer les frais du procès devant la chambre de Police correctionnelle le 9 mars 1848 pour les sévices contre Barthélemy et Elise. CAOM, FM SG MAR, carton 33, dossier 285, Esclavage, sévices. Tableau des affaires de sévices. Affaires. Diverses. Correspondance ministérielle et coloniale, 1844-1848. Extrait des Greffes de la Cour Royale, 1.12.1845, Jean-Louis Simonet de la commune de Robert fut condamné à un an d'emprisonnement, une amende de 50 Francs et les frais du procès pour les sévices contre Laurencin et Maximin. Le cas des frères Jaham est très connu, voir SCHOELCHER: 1847, pp. 297-323; MOITT: 2001, pp. 113-116; SCHMIEDER: 2017, p. 143.

49 http://www.touscreoles.fr/2008/02/20/nous-nous-souvenons/ (25.10.2016). 
Dernièrement, un membre de la famille Marraud des Grottes fit les gros titres de la presse : Vincent Marraud des Grottes tua un Martiniquais noir, Yendi Bamboux âgé de 20 ans, d'un tir dans la tempe. Ce dernier ainsi que ses amis avaient été pris en flagrant délit lors du cambriolage de sa villa dans la commune de François dans la nuit du 9 au 10 mai 2001. Ce n'était pas un acte de légitime défense. Le coup de feu fut qualifié $d^{\prime}$ »accidentel». Vincent Marraud des Grottes ${ }^{50}$ fut acquitté de l'accusation d'homicide devant le Tribunal correctionnel de Fort-de-France en 2003 ainsi qu'en 2004 devant la Cour d'appel de Fort-de France selon I'article 73 du Code de procédure pénale («dans le cas de crime ou de délit flagrant, toute personne a qualité pour en appréhender l'auteur et le conduire devant l'officier de police judiciaire le plus proche»). La Cour de cassation (en Guadeloupe) a cassé le jugement en 2006, les fuyards (qui n'avaient rien volé) n'ayant opposé aucune résistance lors de leur arrestation; ceci atteste l'inutilité de l'usage de la force à leur encontre, laquelle ne pouvait donc être justifiée. ${ }^{51}$ Cependant la Cour d'appel de Basse-Terre rendit son jugement uniquement sur la base des intérêts civils, et Vincent Marraud des Grottes ne fut pas emprisonné pour la mort du jeune homme mais condamné le 4 décembre 2007 à payer $30.000 €$ de dommages et intérêts à la famille de Yendi Bamboux. ${ }^{52}$

Ainsi persistent les conflits sociaux entre les Békés et la majorité afro-descendante de l'île, où les Békés utilisent toujours la force physique avec l'aval de la justice qui continue de soutenir leurs privilèges; ce qui ne signifie pas que tous les membres de la famille Desgrottes ou tous les Békés exploitent les héritages économiques de l'esclavage et perpétuent la tradition de la violence envers la population afro-martiniquaise. Si personne n'est responsable des crimes de ses ancêtres, chacun est toutefois responsable de son propre comportement. Les Békés pourraient répartir les terres entre leurs travailleurs, former une coopérative avec eux ou encore cotiser à un fonds de réparation pour les descendants des esclaves, comme on en discute actuellement (FRITH: 2015; TIN: 2013; FORSDICK: 2015; BECKLES: 2013 pour

50 http://bertrand.auschitzky.free.fr/AppendicesLabarre/MarrauddesGrottes.htm, neuvième génération de la famille Desgrottes.

51 Le Parisien, 30.4.2006, http://www.leparisien.fr/faits-divers/le-meurtrier-d-un-antillais-rejugecinq-ans-apres-les-faits-30-04-2006-2006947955.php (13.6.2016).

52 http://www.lexpress.fr/informations/le-proces-de-toutes-les-passions_725449.html (28.9.2016). 
la Caraïbe anglaise)..$^{53}$ Jusqu'à maintenant, il ne semble pas que beaucoup de Békés aient penché pour ces solutions ou reconnaissent le passé esclavagiste de leurs familles, contrairement à quelques familles esclavagistes du monde anglophone (BALL: 1998; BROWNE: 2008).

J'écris ces lignes, consciente que toute l'Europe a profité directement de la traite et de l'esclavage colonial, les anciennes puissances coloniales tout comme notamment les ports (Liverpool et Bristol, Nantes et Bordeaux, Barcelona et Cádiz, Lisbonne et Porto etc.), ${ }^{54}$ ou indirectement, comme les pays du centre de l'Europe, comme l'Allemagne. ${ }^{55} \mathrm{~A}$ l'occasion de la Décennie internationale des personnes d'ascendance africaine (2014-2015) proclamée par les Nations Unies, il serait bon de se renseigner sur l'origine des richesses des élites européennes et caribéennes. Les désordres du XXe siècle en Europe Occidentale et Centrale et les crimes contre l'humanité plus récents rendent difficile la recherche des descendants des armateurs des navires de la traite, des propriétaires des plantations dans les colonies et des profiteurs du commerce avec les îles esclavagistes en Europe. Cependant, la continuité et l'endogamie des Békés en Martinique permet de trouver les héritiers des richesses accumulées avec le travail des esclaves maltraités.

\section{BibLIOGRAPHIE}

BALL, E. (1998): Slaves in the Family. London, Penguin.

BECKLES, H. (2013): Britain's Black Debt: Reparation for Caribbean Slavery and Native Genocide. Kingston, University of West Indies Press.

BODINIER, J.-L. et BRETEAU, J. (1994): Nantes, un port pour mémoire. Rennes, Éditions Apogée.

53 Lovis-Georges Tin, homme de lettres francais, et Hilary Beckles, historien jamaïcain, sont également des activistes, le premier est président du CRAN (Conseil Représentatif des Associations des Noires de France), le second est président de la commission de réparation des pays caribéens CARICOM. Nicola Frith et Charles Forsdick sont des spécialistes de la littérature et des études postcoloniales qui participent aux discussions sur la réparation, avec quelques sympathies pour la cause de ceux qui demandant des réparations matérielles.

54 Dans le cadre de cet article il est impossible de mentionner toute l'historiographie portant sur ce thème, c'est pourquoi j'indique seulement quelques travaux sur le rôle des ports français et les profits de la traite: DAGET: 1975; BODINIER et BRETEAU: 1994; PÉTRÉ-GRENOUILLEAU: 1998; SAUGERA: 2002.

55 Les Allemands vendaient leurs marchandises - lin, outils - sur les marchés d'Afrique en échange des esclaves ou de la Caraïbe et consommaient le sucre, le café, le cacao, le coton produits par des esclaves (ZEUSKE et LUDWIG: 1995; REIKAT: 1997; WEBER: 2009). 
BROWNE, K. (2008). Traces of the Trade: A Story from the Deep North (documentaire).

CARDIN, J.-L. (1990): La Martinique «chine-chine»: L'immigration chinoise à la Martinique. Paris, L'Harmattan.

BRUNEAU-LATOUCHE, E., CORDIEZ, C. et CORDIEZ, P. (2002): 209 anciennes familles subsistantes de la Martinique. 2 vols., Aix-en-Provence/ Fort-de-France/Paris, E. Bruneau-Latouche.

CAUNA, J. (1997): Haïti: L'éternelle révolution. Histoire d'une décolonisation (1789-1804). Port-au-Prince, Éd. Henri Deschamp.

CHIVALLON, C. (2006): «Rendre visible l'esclavage. Muséographie et hiatus de la mémoire aux Antilles françaises». L'Homme, n 180, pp. 7-42.

COTTIAS, M. (2004): «Droit, justice et dépendance dans les Antilles françaises (1848-1852)». Annales. Histoire, Sciences Sociales, vol. 59:3, pp. 547-567.

COTTIAS, M. (2005): «Et si l'esclavage colonial faisait histoire nationale?». Revue d'Histoire Moderne et Contemporaine, vol. 52:4bis, pp. 59- 63.

DAGET, S. (1975): «Long cours et négriers nantais du trafic illégal, 18141833». Revue Française d'Histoire d'Outre-mer, vol. 62:226-227, pp. 90134.

DESLISLE, P. (1997): Renouveau Missionnaire et société esclavagiste: La Martinique 1815-1848. Paris, Publisud.

FICK, C. E. (1990): The Making of Haiti: The Saint-Domingue Revolution from Below. Knoxville, The University of Tennessee Press.

FORSDICK, C. (2015): "Compensating for the Past: Debating Reparations for Slavery in Contemporary France". Contemporary French and Francophone Studies, vol. 19:4, pp. 420-429.

FRITH, N./ HODGSON, K. (eds.) (2015): At the Limits of Memory: Legacies of Slavery in the Francophone World, Liverpool, Liverpool UP.

FRITH, N. (2015): "Saving the Republic: State Nostalgia and Slavery Reparations in Media and Political Discourses". Modern and Contemporary France, vol. 23:2, pp. 213-232.

HARTKOPF-SCHLOSS, R. (2009): Sweet Liberty: The Final Days of Slavery in Martinique. Philadelphia, University of Pennsylvania Press.

GAUTIER, A. (1985): Les sœurs de solitude: la condition féminine dans l'esclavage aux Antilles du XVIle au XIXe siècle. Paris, Editions Caribéennes.

GEGGUS, D. (1996): «Esclaves et gens de couleur libres de la Martinique pendant l'époque révolutionnaire et napoléonienne: Trois instants de résistance». Revue Historique, CCXCV:1, pp.105-132. 
GEGGUS, D. (1983): «La révolte de Jean Kina à Fort-Royal». Revue de la Société Haïtienne d'Histoire, $\mathrm{n}^{\circ}$ 140, pp. 12-26.

GIRAUD, M.; DUBOST, I.; CALMONT, A.; DANIEL, J.; DESTOUCHES, D. ef MILIA-MARIE-LUCE, M. (2009): «La Guadeloupe et la Martinique dans I'histoire française des migrations en régions de 1848 à nos jours», Hommes et Migrations. Revue Française de Référence sur les Dynamiques Migratoires, $\mathrm{n}^{\circ} 1278$, pp. 174-197.

HIGMAN, B. W. (2000): "The Sugar Revolution", The Economic History Review, New Series, vol. 53:2, pp. 213-236.

JENNINGS, L. (2000): French Anti-Slavery Movement for the Abolition of Slavery in France 1802-1848. Cambridge, Cambridge UP.

LARA, O. D. (2005): La liberté assassinée: Guadeloupe, Guyane, Martinique et la Réunion en 1848-1856. Paris, L'Harmattan.

LARA, O. D. et FISHER-BLANCHET, I. (2011): Propriétaires d'esclaves en 1848. Martinique, Guyane, Saint-Barthélemy, Sénégal. Paris, L'Harmattan. MASSÉ, R. (1980): La fin des plantations?: Évolution des formes de soumission du travail dans deux sociétés néocoloniales: Martinique et Guadeloupe. Ste. Marie, Centre des Recherches Caraïbes.

MICHEL, J. (2015): Devenir descendant d'esclave. Enquête sur les régimes mémoriels. Rennes, Presses Universitaires de Rennes.

MOITT, B. (2001). Women and Slavery in the French Antilles, 1635-1848. Bloomington, Indiana UP.

NICOLAS, A. (1996): Histoire de la Martinique. 2 tomes, Paris, L'Harmattan. NORTHRUP, D. (2000): "Indentured Indians in the French Antilles. Les immigrants indiens engagés aux Antilles Françaises». Revue Française d'Histoire d'Outre-mer, vol. 87:326-327, pp. 245-271.

OUDIN-BASTIDE, C. (2008): Des nègres et des juges. La scandaleuse affaire Spoutourne (183 1-1834). Paris, Editions Complexe.

OUDIN-BASTIDE, C. (2015): Maîtres accusés, esclaves accusateurs: les procès Gosset et Vivié (Martinique, 1848). Mont-Saint-Aignan, Presses Universitaires de Rouen et du Havre.

PAQUET, M.-E. (2009): Regard sur l'économie martiniquaise. Essai. Fort-deFrance, K. Éditions.

PAGO, G. (2006): 1848: chronique de l'abolition de l'esclavage. Fort-deFrance, Desnel.

PAGO, G. (2011): L'insurrection de Martinique, 1870-1871. Paris, Syllepse. PÉTRÉ-GRENOUILLEAU, O. (1998): Nantes au temps de la traite des Noirs, Paris, Hachette. 
POPKIN, J. (2010): You are all free: The Haitian Revolution and the Abolition of Slavery. Cambridge, Cambridge UP.

POTTHAST B. (1998): "The Slave Family in the Caribbean: A Research Review". Ibero-Amerikanisches Archiv. vol. 24:3-4, pp. 279-301.

REINHARDT, C. A. (2006): Claims to Memory, Beyond Slavery and Emancipation in the French Caribbean. New York/ Oxford, Berghahn Books.

REIKAT, A. (1997): Handelsstoffe. Grundzüge des europäisch-westafrikanischen Handels vor der Industriellen Revolution am Beispiel der Textilien. Cologne, Rüdiger Köppe Verlag.

RENARD, R. (1993): "Labour Relations in Martinique and Guadeloupe, 1848-1870", à BECKLES, H./ SHEPHERD, V. (eds.), Caribbean Freedom: Economy and Society from Emancipation to the Present, A Student Reader. London, James Currey, Kingston, lan Randle, pp. 80-92.

SAUGERA, E. (2002) Bordeaux: Port négrier, chronologie, économie, idéologie, XVIle-XIXe siècles. Paris, Karthala.

SCHMIDT, N. (2009): La France a-t-elle aboli l'esclavage? Guadeloupe Martinique - Guyane (1830-1935). Paris, Perrin.

SCHMIEDER, U. (2014 a): "(Antiguos/as) esclavizados/as como padres y madres: Martinica y Cuba comparadas". Revista Cuadernos del Caribe, vol. 18:2, pp. 21-35.

SCHMIEDER, U. (2012): "Conflictos sociales en plantaciones martiniquesas antes y después de la abolición de la esclavitud". Revue de la Société Haïtienne d'Histoire, de Géographie et de Géologie, n² 245-248, pp. 142-181. SCHMIEDER, U. (2017): Nach der Sklaverei, Martinique und Kuba im Vergleich, Berlin, LIT.

SCHMIEDER, U. (2014 b): "Sexual relations between the Enslaved and between Slaves and Non-slaves in Nineteenth Century Cuba", à CAMPBELL, G. et ELBOURNE, E. (eds.), Sex, Power and Slavery. Athens, Ohio UP, pp. 227-252.

SCHMIEDER, U. (2013): "The Teaching Religious Orders and Slave Emancipation in Martinique". Journal of Caribbean History, vol. 47:2, pp. 153-183.

SCHOELCHER, V. (1847): Histoire de l'esclavage pendant les deux dernières années. Paris, Pagnerre.

SMÉRALDA-AMON, J. (1996) : La question de l'immigration indienne dans son environnement socio-économique martiniquais 1848-1900. Paris/ Montréal, L'Harmattan.

SMÉRALDA, J. (2009): Guadeloupe, Martinique, des sociétés en révolte: morphologie d'un conflit social. Fort-de-France (Martinique), K. Editions. 
THESÉE, F. (1993): «La révolte des esclaves du Carbet à la Martinique (1822)». Revue Française d'Histoire d'Outre-mer, vol. 80:301, pp. 551584.

TIN, L.-G. (2013): Esclavages et réparations. Comment faire face aux crimes de l'histoire. Paris, Stock.

TOMICH, D. (1990): Slavery in the Circuit of Sugar: Martinique and the World Economy, 1830-1848, Baltimore, Johns Hopkins UP.

TOMICH, D. (1995): "Contested Terrains: Houses, Provision Grounds and the Reconstitution of Labour in Post-Emancipation Martinique", à TURNER, M. (ed.), From Chattel Slaves to Wages Slaves: The Dynamics of Labor Bargaining in the Americas. Bloomington and Indianapolis, Indiana UP, pp. 241-257.

TOMICH, D. (1990):"'Liberté ou Mort': Republicanism and Slave Revolt in Martinique 1831, History Workshop Journal, $\mathrm{n}^{\circ} 29$, pp. 85-91.

WEBER, K. (2009), «Deutschland, der atlantische Sklavenhandel und die Plantagenwirtschaft der Neuen Welt (15.-19. Jahrhundert)". Journal of Modern European History, vol. 7:2, pp. 37-67.

ZEUSKE, M. et LUDWIG, J. (1995): «Amerikanische Kolonialwaren in Preußen und Sachsen: Prolegomena". Jahrbuch für Geschichte von Staat, Wirtschaft und Gesellschaft Lateinamerikas, vol. 32, pp. 257-301. 